\title{
Research Progress of Stress-Induced Magnetic Anisotropy in Fe-Based Amorphous and Nanocrystalline Alloys
}

\author{
Raymond Kwesi Nutor ${ }^{1,2}$, Xiaozhen Fan ${ }^{1,2}$, Sensheng Ren ${ }^{1,2}$, Ming Chen ${ }^{1,2}$, Yunzhang Fang1,2* \\ ${ }^{1}$ Condensed Matter Physics Laboratory, Zhejiang Normal University, Jinhua, China \\ ${ }^{2}$ State Key Laboratory of Solid State Photo-Electronic Devices, Zhejiang Normal University, Jinhua, China \\ Email:^fyz@zjnu.cn
}

How to cite this paper: Nutor, R.K., Fan, X.Z., Ren, S.S., Chen, M. and Fang, Y.Z. (2017) Research Progress of Stress-Induced Magnetic Anisotropy in Fe-Based Amorphous and Nanocrystalline Alloys. Journal of Electromagnetic Analysis and Applications, 9, 53-72.

https://doi.org/10.4236/jemaa.2017.94006

Received: April 11, 2017

Accepted: May 13, 2017

Published: May 16, 2017

Copyright $\odot 2017$ by authors and ScientificResearch Publishing Inc.

This work is licensed under the

CreativeCommons Attribution

International

License (CC BY 4.0).

http://creativecommons.org/licenses/by/4.0/

\begin{abstract}
Since it was discovered that stress annealing induced larger anisotropies compared to other annealing methods in amorphous and nanocrystalline alloys, there has been a lot of research done to explain this phenomenon. This has led to many suggestions about the origin of this stress-induced magnetic anisotropy, but till now the origin is explained with two competing models: the magnetoelastic effect model and the diatomic pair ordering model. In spite of these theories, the origin of the stress-induced anisotropy is still under discussion because direct observation of structural anisotropy is still lacking. In this paper, we have reviewed some of the characterization techniques which have been used to discuss the origin of stress-induced magnetic anisotropy and the progress which has been made thus far in unifying all the contrasting views which has been suggested to be the origin of the stress-induced anisotropy in FINEMET alloys.
\end{abstract}

\section{Keywords}

Magnetic Anisotropy, FINEMET Alloys, Stress Annealing

\section{Introduction}

On the macroscopic level, a magnet is labelled by its north and south poles, however on the microscopic level, magnetism is dependent on whether a material is crystalline or non-crystalline [1]. If a material is crystalline, then the magnetic properties becomes directional, this means that for a specific magnetic material the magnetic moments which contribute to the material's magnetic property align themselves preferentially, in what is called the "easy axis" [2]. This phenomenon is known as magnetic anisotropy. In the high frequency range, a large anisotropy is needed to be able to modify the magnetic properties of a ma- 
terial to suit different applications, therefore, it is important to understand its origin. One of the most important magnetic materials is the ferromagnetic materials and in this article, we will narrow our study down to the FeCuNbSiB ferromagnetic alloys since they have gained significant attention in recent times. Altering the composition of a magnetic material affects its magnetic properties such as the hysteresis loop, permeability, anisotropy and magnetic flux. Also, whether the ferromagnetic material is crystalline or amorphous plays an important role.

Nanocrystalline alloys produced by the partial crystallization of FeCuNbSiB amorphous alloys are one of the best known soft magnetic alloys [3] [4]. These alloys possess excellentpermeability while maintaining a high saturation magnetization, low coercivity, high electrical resistivity, high Curie temperatures and low energy losses all of which make them suitable candidates for most industrial and technological applications [4] [5] [6]. Being one of the pioneers of soft nanocrystalline alloys, a lot of research has been done on the FeCuNbSiB alloy most of which is geared towards tailoring the properties to suit a specific application. Some of the methods which have been used to tailor these properties are annealing which is microstructural modification process [7] and addition of other alloying elements such as $\mathrm{Co}, \mathrm{Ni}, \mathrm{Mo}, \mathrm{Zr}, \mathrm{Ta}, \mathrm{Cr}$. Quite recently, $\mathrm{Cr}$ containing nanocrystalline have attracted some interest [8] [9] due to the positive effect chromium has on soft magnetic properties in addition to corrosion resistance and increase in thermal stability [10] whilst showing high values of stress induced magnetic anisotropy constant. Induced anisotropy was shown to be a special way in tailoring magnetic permeability by stress or field annealing [11] [12] of which stress annealing was found to induce the highest anisotropy in soft nanocrystalline alloys compared. Since its discovery [3], the unique dual-phase microstructure of the $\mathrm{FeCuNbSiB}$ nanocrystalline alloys has been attributed to its possessing excellent magnetic properties and even to an extent, good corrosion properties. In obtaining the Fe-based soft nanocrystalline alloys, its amorphous precursors are annealed at temperatures between $520^{\circ} \mathrm{C}-580^{\circ} \mathrm{C}$ [13], although recent works show $540^{\circ} \mathrm{C}$ is the nanocrystallization temperature. After the amorphous alloys have been annealed they possess a two-ferromagnetic phase microstructure of nanocrystalline grains dispersed or surrounded in an amorphous matrix.

Knowledge of these magnetic properties are helping develop state of the art magnetic sensors and data storage materials which play a key role in modern technology. This paper aims to provide a summary on the contributions which have been done to enhance the understanding of stress-induced anisotropy in amorphous and nanocrystalline alloys. The formatter will need to create these components, incorporating the applicable criteria that follow.

\section{Fundamentals of Magnetic Anisotropy}

\subsection{What is Magnetic Anisotropy}

When a material is said to be anisotropic, it means its properties are direc- 
tion-dependent. Therefore, magnetic anisotropy is simply the dependence of magnetic properties on direction. Ferromagnetic materials are seen to be the most important type of magnetic materials and it is in them that this magnetic anisotropy phenomenon happens [1] [2]. Magnetization in ferromagnetic materials results from contributions to its magnetic energy. Domains are small volume regions found in ferromagnetic materials which contains magnetic dipole moments and it is the alignment of these dipole moments which determine the degree of magnetization [14]. For example, if an external magnetic field is applied to a ferromagnetic material and the magnetic dipole moments in the domains are aligned in the same direction as the external magnetic field it is said that saturation magnetization has occurred [1] [2]. The basic principle is that ferromagnetic particles have various contributions to the magnetic energy which controls their magnetization. No matter how simple or complex the combination of energies maybecome, the grain will seek the configuration of magnetization which minimizes its total energy [15]. The short answer to our question is that certain directions within magnetic crystals are at lower energy than others. To shift the magnetization from one "easy" direction to another requires energy. If the barrier is high enough, the particle will stay magnetized in the same direction for very long periods of time [15] [16]. We have now seen that magnetic anisotropy is mostly dependent of the domain structure of the ferromagnetic materials, however, the type of magnetic anisotropy is dependent on (a) crystal structure (b) grain shape and (c) residual stress [17]. Now, let us have it in mind that all of these affect the hysteresis loop or behaviour, the coercivity and the remanence of the material.

\subsubsection{Magnetocrystalline Anisotropy}

When the energy required to magnetize a material varies with respect to crystallographic directions it is known as magnetocrystalline anisotropy [2]. The crystallographic direction which requires the minimum amount of energy to magnetize the material is known as the easy axis whilst the direction which requires the maximum amount of energy to cause magnetization is known as the hard axis. Spin-orbit coupling is the main source of magnetocrystalline anisotropy [14].

We see in Figure 1, that for both metals a different curve was generated when the magnetic field was applied in each of [100], [110] and [111] crystallographic directions. Since most metals have a cubic structure, let's look at the magnetocrystalline energy in cubic materials. In cubic materials, the magnetocrystalline energy density is given by:

$$
\epsilon_{v}=K_{1}\left(\alpha^{2} \beta^{2}+\beta^{2} \gamma^{2}+\gamma^{2} \alpha^{2}\right)+K_{2} \alpha^{2} \beta^{2} \gamma^{2}
$$

where $K_{1}$ and $K_{2}$ are magnetocrystalline anisotropy constants, $\alpha, \beta, \gamma$ are the directions.

\subsubsection{Shape Anisotropy}

This is due to the shape of the grain of the material [18]. When a body is magne- 


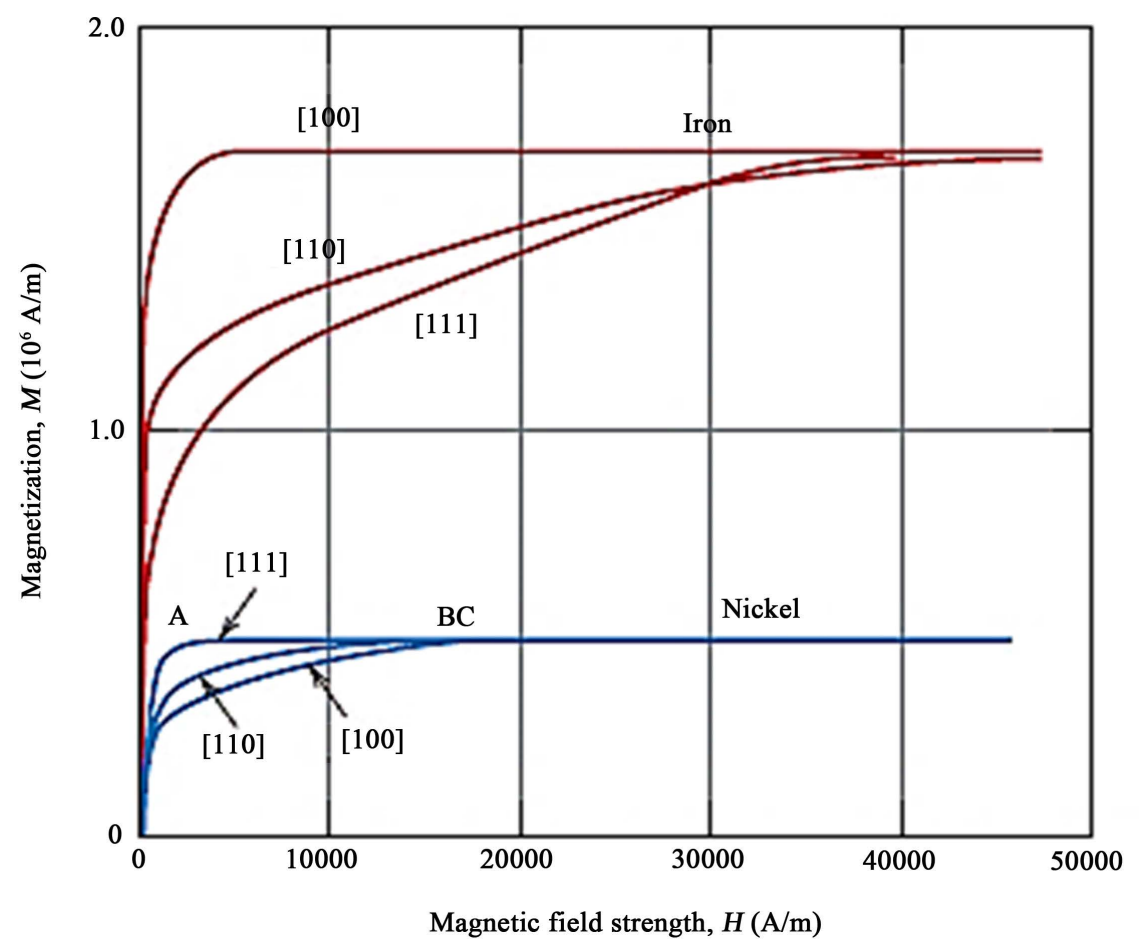

Figure 1. The magnetization curves for iron and nickel [2].

tized it produces magnetic charges at the surface, which we call "poles". This surface charge distribution is a source of magnetic field and it is known as the demagnetizing field since it acts in opposition to the magnetic field which produces it [19]. The unique feature of permanent magnets is their ability to store magnetostatic energy and their shapes were dictated by the low coercivity $\mathrm{H}_{\mathrm{c}}$ of the ferromagnetic materials available over a 100 years ago, which limited the tolerable demagnetizing field in the second quadrant of the $\mathrm{M}(\mathrm{H})$ hysteresis loop where the working point of a magnet is inevitably located [20].

This demagnetizing field is proportional to the magnetization of the material and is sensitive to shape [21] [22]. The demagnetizing field is expressed as:

$$
H_{d}=-N M
$$

where $H_{d}$ is the demagnetizing field, $M$ is the magnetization and $N$ is the demagnetization factor determined by shape. Shape anisotropy is only fully effective in regions where the magnetization remains uniform and rotates coherently without breaking upinto domains [20].

\subsubsection{Stress Anisotropy}

This kind of anisotropy can be said to be the most common in ferromagnetic materials. This is because most manufacturing or processing techniques leave residual stresses or strains in a material which affects atomic interactions, which therefore affects spin-orbit interactions which generally gives rise to magnetic energy [23]. Changes in magnetization can change the shape of the crystal by altering the shapes of the orbitals [24]. This phenomenon is called magnetostriction. This is to say that a magnetic material will change its dimension when 
magnetized [25]. The inverse effect also occurs; the magnetic behaviour of a material varies with applied stress [26].

The magnetic anisotropy energy is related to stress as:

$$
K_{u}=-\frac{3}{2} \sigma \lambda_{s}
$$

where $\sigma$ is the applied or residual stress and $\lambda_{s}$ is the saturation magnetostriction constant. Figure 2 shows the relationship between induced anisotropy energy and applied stress during annealing as shown by Alves et al. [27].

Since stress is related to strain, sometimes the magnetic anisotropy energy is rather related to the strain [26]. The effect of induced anisotropy on magnetic hysteresis properties like remanence and coercivity is explained by the back-stress model [28]. Per the back-stress model, the effective permeability and the uniaxial magnetic anisotropy energy are related as follows:

$$
\frac{1}{\mu_{\text {eff }}}=\frac{2 \mu_{o} K_{u}}{J_{s}^{2}}
$$

where $\mu_{o}$ is the vacuum permeability, $\mu_{\text {eff }}$ is the effective permeability and $\mathrm{J}_{\mathrm{s}}$ is the saturation magnetization. The inverse relationship between effective permeability and the uniaxial magnetic anisotropy of FINEMET type alloys seen in the work done Csizmadia et al. [29] is shown in Figure 3.

\section{Characterization Techniques of Magnetic Materials}

When a material is fabricated in the lab, how are we able to determine that our method was successful? Depending on the nature of the material being investigated, a group of techniques may be utilized to assess its structure and properties. Whereas some techniques are qualitative, such as providing an image of a surface, others yield quantitative information such as relative concentrations ofatoms that compromise the material [30]. However, our main focus is to look at some of the methods used to characterize magnetic materials.

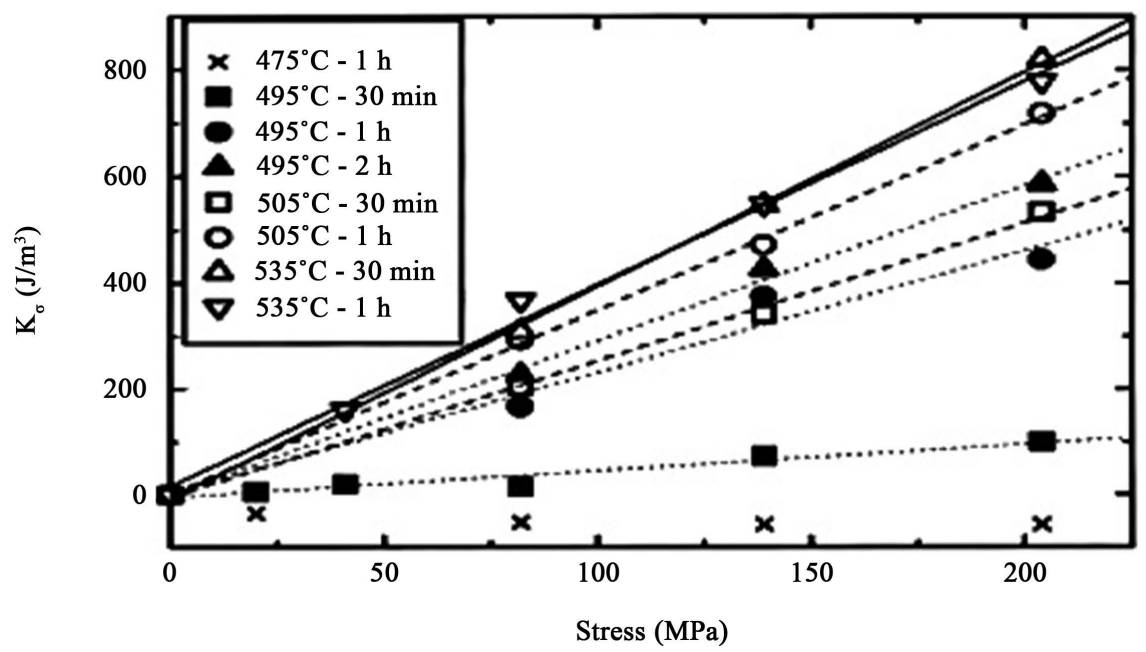

Figure 2. Relationship between induced anisotropy and applied stress during annealing treatment of FeCuNbSiB alloy shown by Alves et al. [27]. 


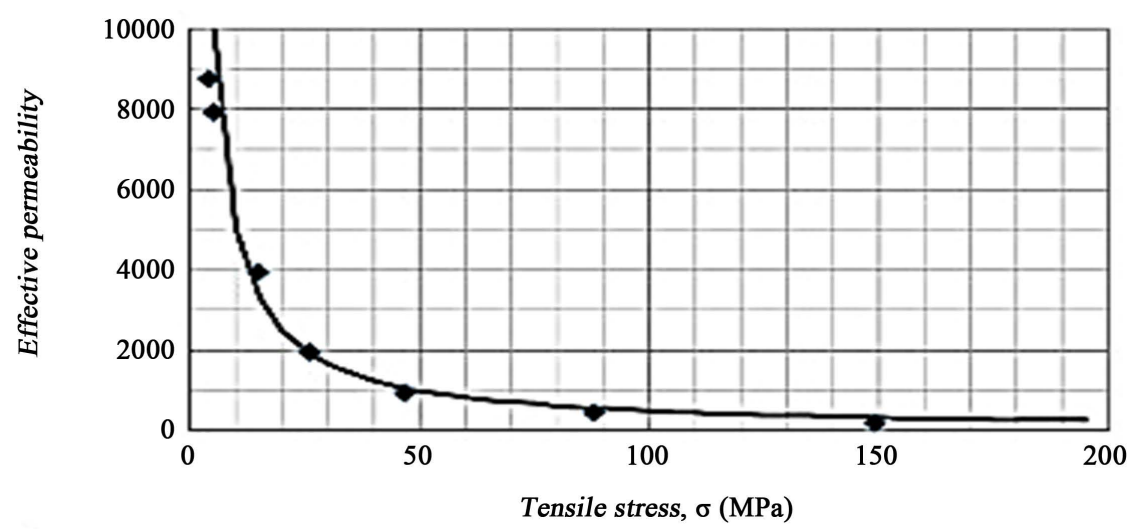

(a)

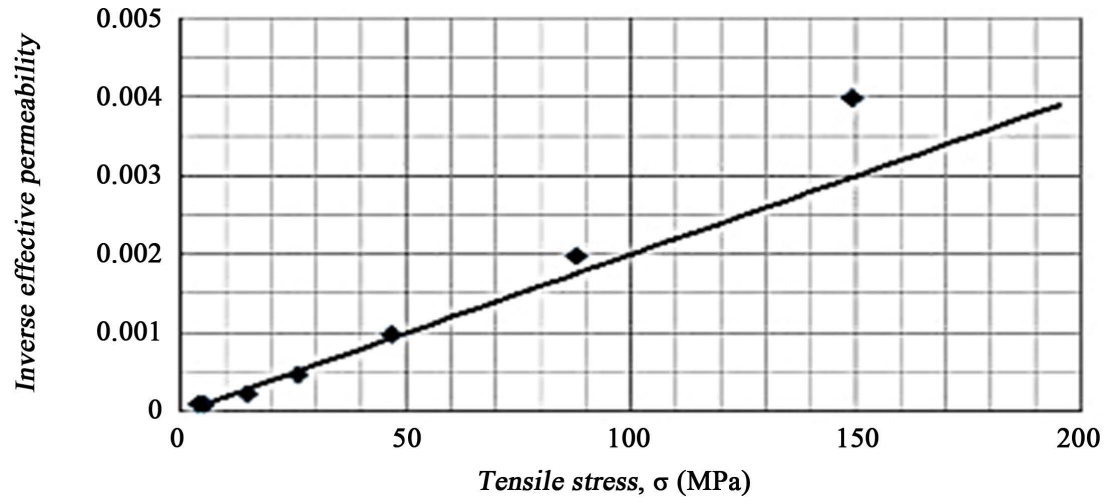

(b)

Figure 3. Effective permeability (a) and inverse effective permeability (b) vs. applied stress in FINEMET-type ribbons as shown by Csizmadia et al. [29].

The structure of magnetic materials can be divided into two parts; physical structure and magnetic structure [31]. The physical structure comprises of the grain size, grain boundaries, crystal structure, morphology, texture, volume fraction of each phase, surfaces, defects,inter-phase interfaces, etc. whilst the magnetic structure consists of magnetic-domain structure and recording pattern in magnetic recording media [31] [32].

\subsection{X-Ray Diffraction}

This can be used to identify the phases present in a material and to provide information on the physical state of the sample, such as grain size, texture and crystal perfection. However, since we're dealing with characterizing magnetic materials, information from XRD will tell us whether a material is either amorphous or crystalline. The peaks from XRD data also gives information about the isotropic or anisotropic nature of a magnetic material before and after it has been subjected to a heat treatment process [33]. Therefore, XRD is one of the most common material characterization techniques. X-rays are a form of electromagnetic radiation that have high energies and short wavelengths-wavelengths on the order of the atomic spacing for solids. When a beam of $\mathrm{x}$-rays impinges on a solid material, a portion of this beam will be scattered in all direc- 
tions by the electrons associated with each atom or ion that lies within the beam's path [34]. A simple expression which relates the wavelength of the $\mathrm{x}$-ray and the interatomic spacing to the angle of the diffracted beam known as the Bragg's law is important condition which needs to be satisfied during diffraction. If the Bragg's law is not satisfied, then the interference will be nonconstructive, therefore, yielding a very low-intensity diffracted beam [2] [30].

Because we are concerned about identifying the phases present in the soft nanocrystalline materials after their amorphous precursors have been annealed, we look at some previous work which have used x-ray diffraction methods to characterize magnetic materials. The amorphous nature of as-cast or prepared ribbons have been confirmed using XRD measurements by most researchers and different phases have also been identified depending on the composition, annealing temperature and time. Quite recently, Gavrilovic et al. [35] identified multiple crystalline phases in a FeCuNbSiB sample which was heat treated at $1123 \mathrm{~K}$ for $24 \mathrm{~h}$. Kurlyandskaya et al. [10] used the XRD to analyze the texture features in soft nanocrystalline $\mathrm{FeCrSiBNbCu}$ alloys and for different compositions it was shown that stress annealing typically results in no texturing [36]. Figure 4 shows the XRD patterns of an FeCuNbSiB sample annealed at 973K studied by Gavrilovic et al. [37] which revealed the different phases which become present after the sample has been subjected to the annealing process.

Therefore, we can see that structural information of nanocrystalline and amorphous ribbons can be obtained by conventional XRD. However, the information obtained from conventional XRD is not enough to be able to clarify in details the origin of stress-induced magnetic anisotropy.

\subsection{Transmission X-Ray Diffraction}

In this type of diffraction technique, the sample is subjected to an incident $\mathrm{x}$-ray beam which passes through the material, interacts with the atoms and is diffracted. Details of the interaction is captured by a detector. Because, we want the

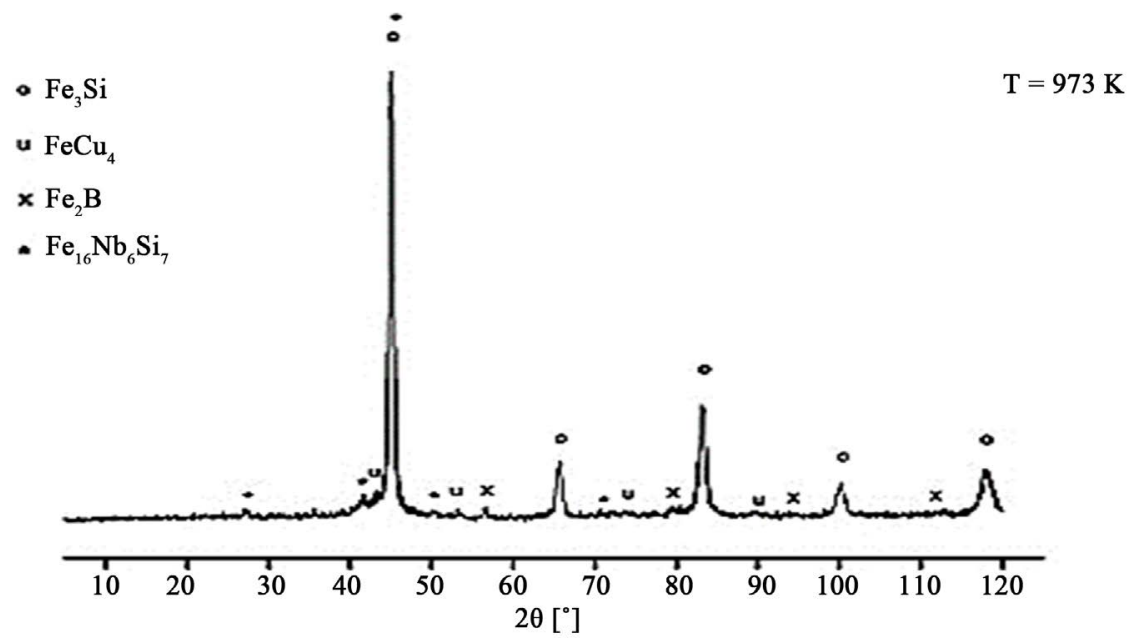

Figure 4. XRD diffractogram of FeCuNbSiB sample annealed at $973 \mathrm{~K}$ for one hour by Gavrilovic et al. [37]. 
information obtained to be accurate enough, samples are normally subjected to incident $\mathrm{x}$-ray beams at different angles which results in different diffraction angles [34]. The most common $\mathrm{x}$-ray radiation source used for magnetic materials is $\mathrm{Mo}-\mathrm{Ka}$ radiation. A monochromator is also used to select a narrow band of wavelength of radiation or light from a wider range of wavelengths [33]. X-ray diffraction in transmission mode has been used to study structural and magnetic anisotropy in both nanocrystalline and amorphous alloys. Now, let's look at some of them.

The stress induced magnetic and structural anisotropy of nanocrystalline Fe-based alloys was studied using the XRD in transmission mode by Ohnuma et al. [11]. The samples were amorphous ribbons of $\mathrm{FeSiBNbCu}$ with different elemental compositions which were subjected to annealing treatments with/without varying applied stresses. The XRD measurements taken for each sample were in two modes: (a) the diffraction vector parallel to the ribbon direction (b) the diffraction vector perpendicular to the ribbon direction. Information obtained from the data showed the presence of structural anisotropy in the alloys as shown in Figure 5.

The mechanism of the magnetic anisotropy in the nanocrystalline alloys annealed under stress was investigated using $\mathrm{x}$-ray diffraction in transmission mode by Ohnuma et al. [12]. Two profiles were measured with diffraction vectors parallel and perpendicular to the ribbon direction. The XRD profiles of the alloy annealed under tensile stress showed a clear difference between the two directions. The Induced magnetic anisotropy in Si-free nanocrystalline soft magnetic materials was studied via transmission x-ray diffraction by Parsons et al. [26]. In this experiment, all samples underwent two measurements, one was

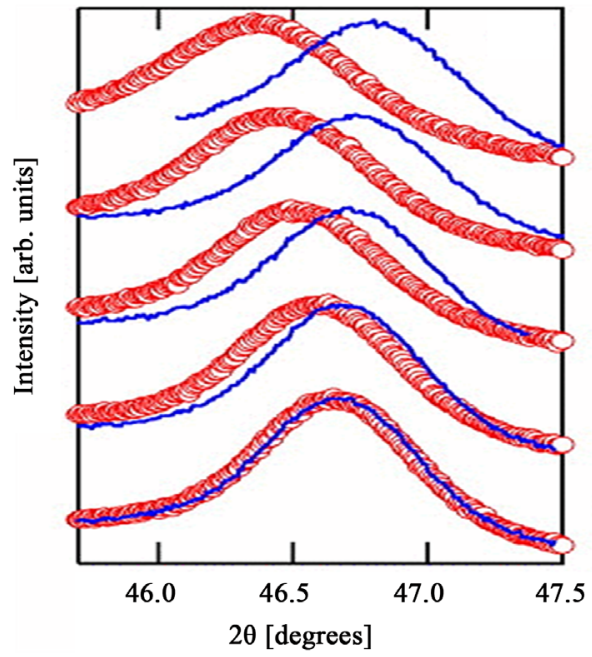

(a)

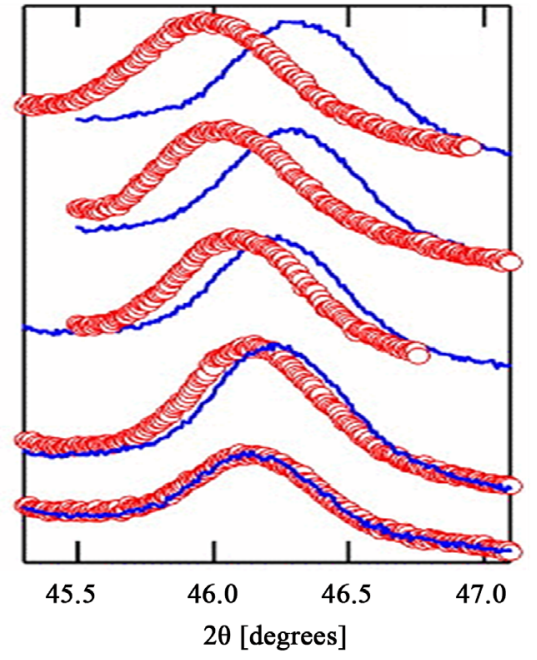

(b)

Figure 5. t-XRD profiles for (a) (620) plane of phase in and (b) (310) plane of Bcc-Fe phase in alloys annealed under different tensile stress. Circles and curves indicate t-XRD profiles with diffraction vector parallel and perpendicular to the applied stress, respectively. The applied stress is (a) 10,102, 212, 332, and $469 \mathrm{MPa}$, (b) 10, 102, 207, 313, and $433 \mathrm{MPa}$, from bottom to top [11]. 
taken parallel and the other was taken in the perpendicular direction, relative to the applied field placed on the sample during the annealing process. When a Gaussian function was fitted to the reflection peaks, a small but consistent, change in peak position was found in the parallel and perpendicular directions in which the measurements were taken. Ohnuma et al. [38] studied the effect of applied stress on the structural anisotropy using transmission XRD and they found that the lattice spacing of certain planes shows elongation (anisotropy) proportional to the applied stress. In the study of the structural anisotropy of amorphous alloys with creep induced magnetic anisotropy by Ohnuma et al. [39], transmission XRD measurements showed that samples annealed under stress reveal a structural anisotropy which is released post-annealing as a residual strain and this strain increases with annealing stress which in turn is also related to the magnetic anisotropy.

The transmission XRD has been a valuable tool in the quest to understand the origin of the stress induced magnetic anisotropy. It has been used to verify the magnetoelastic model and at the same time show that the contribution of the diatomic pair ordering to the stress-induced magnetic anisotropy is quite negligible.

\subsection{Transmission Electron Microscopy}

Transmission electron microscopy is a very important and powerful tool in characterizing the structure of magnetic materials primarily due to its high sensitivity, enabling simultaneous examination of microstructural features through high-resolution imaging and acquisition of chemical and crystallographic information from small regions of the specimen [31]. The Transmission electron is built around an electron column which can be divided into two main assemblies: the electron gun-which is the source of high energy electrons and electromagnetic lenses-which is used to control the beam and thus generate the image [31]. There are different types of interactions which occur when the electrons interact with the sample. One of these interactions, diffraction of the electrons by the periodic array of atomic planes in the specimen, ultimately produces the contrast that most commonly enables observation of structural details in crystalline materials [40]. The electrons that pass through the thin crystal without being diffracted by the atomic planes are referred to as transmitted electrons. The electrons that have been diffracted from a given (hkl) plane are the (hkl) diffracted electrons. Contrast in amorphous materials is primarily due to electron absorption effects, which increase with atomic number. Ultimately, an area of interest is illuminated due to the flooding of sample with electrons [31].

Because the Transmission electron microscope can be used in both image mode and diffraction mode or combined diffraction and image mode, both structural and morphological information can be obtained therefore it can be used to characterize magnetic materials with precision. Figure 6 shows typical XRD patterns of stress and field annealed samples.

The structural origin of stress-induced magnetic anisotropy in $\mathrm{FeSiBNbCu}$ 


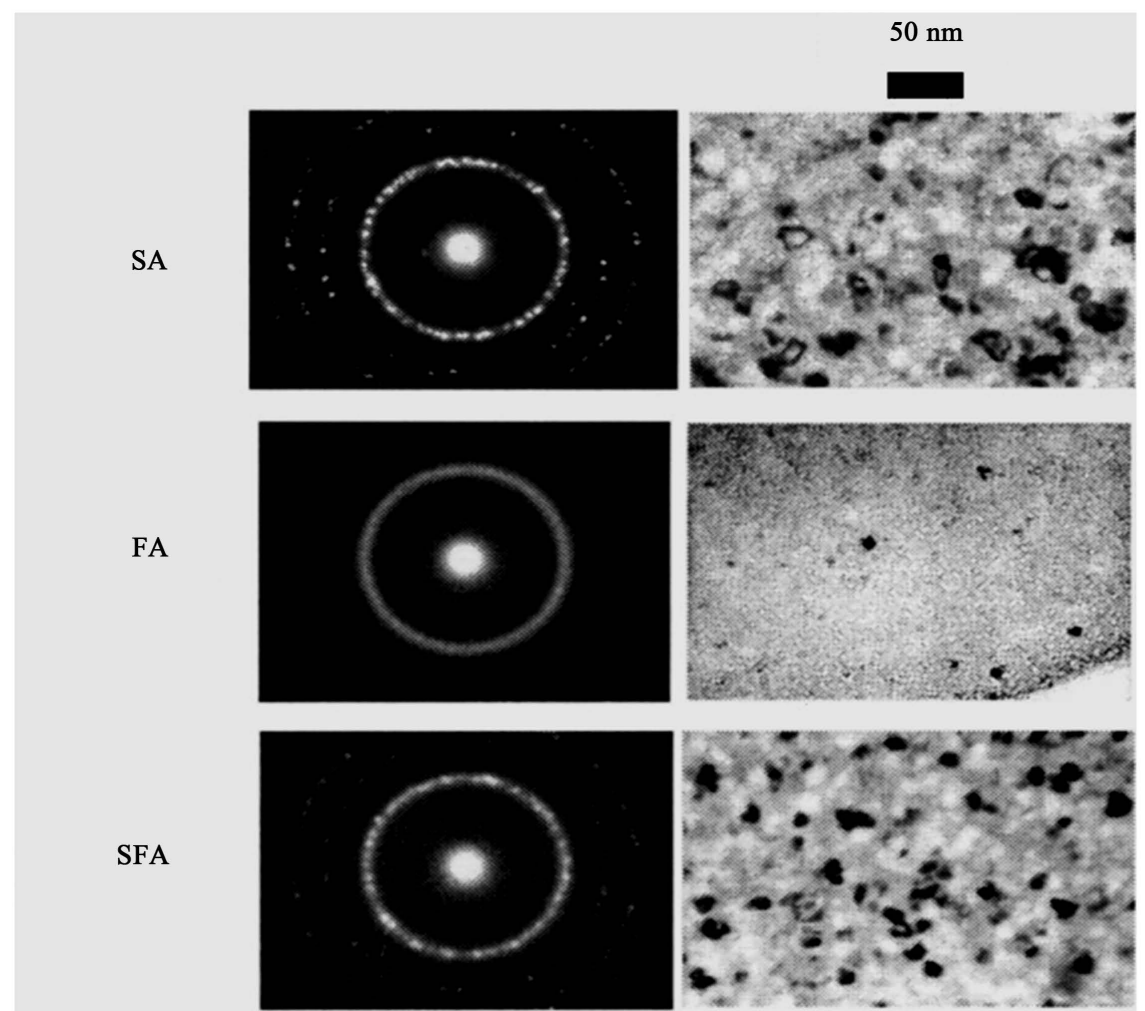

Figure 6. TEM patterns of samples stress annealed (SA), field annealed (FA) and Stress and Field annealed (SFA) shown by Miguel et al. [38].

nanocrystalline alloys was studied using TEM. In this experiment TEM observations was carried out on the samples annealed under stress to check the texture and shape of the anisotropy [12]. However, the TEM revealed isotropic profiles which was as indication of the absence of shape anisotropy and texture in contrast to the results obtained using the transmission XRD. The microstructural character of CoSiBFeNb amorphous alloys under the effect of stress and/or field annealing was studied using TEM analysis and information obtained was able to clarify the microscopic mechanisms responsible for the magnetic anisotropy induced by the different thermal treatments of the samples [41].

The low temperature magnetic behaviour of glass-covered magnetic microwires with gradient nanocrystalline microstructure has been studied using Transmission Electron Microscopy. TEM observations indicated how crystallization occurred at different temperature ranges [42].

Unlike XRD techniques, the TEM provides more precise information about grain size distribution and the microstructure, although the Scherrer equation can use information obtained from the XRD to estimate the grain size of samples. The TEM reveals the unique composite microstructure of $\mathrm{Fe}(\mathrm{Si})$ nanocrystals dispersed in a residual amorphous matrix for amorphous ferromagnetic materials during devitrification.

\subsection{Mössbauer Spectroscopy}

Mössbauer spectroscopy is a characterization technique based on the Mössbauer 
effect which was discovered by Rudolf Mössbauer in 1958. The technique is based on the phenomenon of the emission and resonant absorption and scattering of nuclear gamma rays without energy loss due to recoil. In the spectroscopy process, a solid sample is exposed to a beam of gamma radiation and a detector measures the beam intensity transmitted through the sample. It is very important that the atoms in the gamma ray emitting source be of the same isotope as the atoms absorbing them [43]. Since the demand for efficient magnetic materials for technological applications have increased over the years, a lot of research has/is being done to improve required properties of the magnetic materials to meet the increasing scientific and technological demands [44]. Therefore, Mössbauer spectroscopy which provides delicate details about the properties of magnetic materials is a very important tool in the study of magnetic materials.

We have seen over the years that the scale of materials has evolved from a large scale to a small scale while still maintaining or even improving on their properties. Today, most materials have been produced on the nanometer level which we call nanomaterials. The Mössbauer spectroscopy can distinguish atomically, magnetically or electronically in-equivalent lattice sites and provides information about local neighborhood of certain probe sites. Because, nanocrystalline materials exhibit superior soft magnetic properties, it provides us the opportunity to study how these properties are related to grain refinement on the nano scale [45].

The local field-induced anisotropy of the residual amorphous phase and the bcc crystalline phase was determined using Mössbauer spectroscopy where it was found that with increasing volume fraction of the crystalline phase the field-induced anisotropy increased [26]. In the study of the Induced magnetic anisotropy and structure of Fe-based nanocrystalline materials with different $\mathrm{Si}$ and Co content, Lukshina et al. [46] used both Mössbauer spectroscopy and high-resolution electron microscopy for structural investigation and the correlation between the phase composition of the nanocrystalline materials with the type of induced magnetic anisotropy and thermal stability of magnetic properties after stress annealing was established [46]. Kai-Yuan He [47] used Mössbauer spectrum measurements to determine the specific magnetization of the residual amorphous phase in the study of the optimum condition for magnetic properties of two-phase soft magnetic alloys. Mössbauer, magnetic and XRD measurements showed that the anisotropic atomic rearrangements associated with stress annealing, induces magnetic anisotropy in a plane perpendicular to the stress axis in the work done by Kane et al. [48]. In the Mössbauer study of FeCuNbSiB-type nanocrystals, it was seen that with increasing annealing time, there was an increase in the number of $\mathrm{Fe}$ atoms with $\mathrm{Nb}$ and $\mathrm{Cu}$ as the nearest neighbor atoms, resulting in a decrease in the average value of hyperfine magnetic field distribution in the residual amorphous phase [49]. Mössbauer measurements was performed by Gupta et al. [50] to obtain additional information about the redistribution of the elements in a $\mathrm{FeCuNbSiB}$ sample which was an- 
nealed at $400^{\circ} \mathrm{C}$ with varying annealing times in the study of the $\mathrm{Cu}$ clustering kinetics in the amorphous phase of the FeCuNbSiB alloy. The Mössbauer spectra analysis by Hasiak et al. [51] found that the volume fraction of the crystalline $\alpha$-FeSi phase equaled $8 \%$ and the iron content of the crystalline $\alpha$-FeSi phase was the same as that of the amorphous matrix for the FeCuNbSiB samples annealed at $733 \mathrm{~K}$ for 4 hours. However, the amount of crystalline $\alpha-\mathrm{FeSi}$ phase increased with annealing time, with the volume fraction of the $\alpha-\mathrm{FeSi}$ crystalline phase equaling $25 \%$ after annealing at $733 \mathrm{~K}$ for 14 hours.

\section{The Origin of Magnetic Anisotropy}

$\mathrm{FeCuNbSiB}$ soft nanocrystalline alloys have a wide range of industrial applications, and in high frequency ranges, high induced anisotropy and high electrical resistivity are necessary to improve the properties. It has been seen over the years that the induced anisotropy in soft nanocrystalline alloys can be altered by partial substitution of Fe content in FINEMET type alloys with Co or Ni [52] [53] of which the relative permeability can be reduced from about 20000 to 2500 [54] or the application of stress or magnetic field. Having been reported first in the 90s [55] [56] it has been found that stress annealing induces anisotropy values of over $3000 \mathrm{~A} / \mathrm{m}$ which is far larger compared to anisotropy values obtained by conventional magnetic field annealing processes [28] [36] [57]. During the annealing process of the $\mathrm{FeCuNbSiB}$ alloys, $\mathrm{Cu}$ clusters appear in the amorphous matrix, the $\mathrm{Cu}$ clusters increases eventually into a FCC-Cu which acts as heterogeneous nucleation sites for $\alpha$-Fe crystals from which $\mathrm{Nb}$ and $\mathrm{B}$ are rejected [5] [7] [58] [59] [60] [61]. The rejected solutes stabilize the remaining amorphous phase, retarding the further growth of the $\alpha$-Fe nanocrystals [62] [63]. Nb is also responsible for the thermal stability of the nanocrystalline size [64]. Si partitions grow in the $\alpha$-Fe phase, thereby ending up with the $\mathrm{DO}_{3}$ structure. The concentration of $\mathrm{Si}$ in the $\alpha$-Fe phase has been estimated to be approximately 20 at $\%$ in earlier investigations [61] [65] [66]. The microstructure produced after the annealing process is a composite of $\alpha-\mathrm{Fe}(\mathrm{Si})$ nanograins dispersed in a residual amorphous matrix. The amorphous to nanocrystalline phase transformation during the annealing process is shown in Figure 7. The $\alpha-\mathrm{Fe}(\mathrm{Si})$ are exchange coupled through the thin ferromagnetic amorphous phase because the grain size

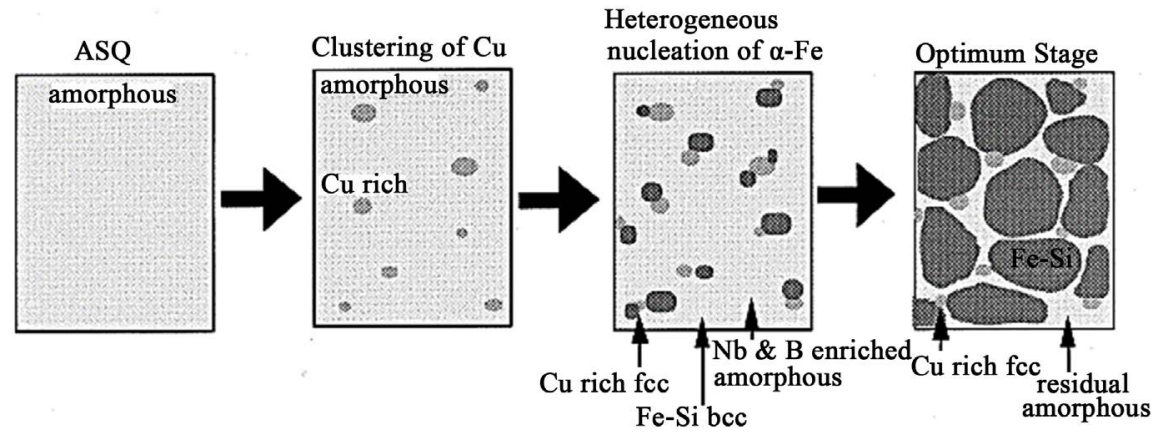

Figure 7. Schematic drawing of the microstructural evolution of $\mathrm{FeCuNbSiB}$ amorphous samples during primary crystallization as studied by Hono et al. [13]. 
is small. The coexistence the nanocrystalline and amorphous phases yields a good balance between positive and negative saturation magnetostriction values which results in ultra-soft magnetic properties.

With the origin of stress induced anisotropy, previous work has led to the proposal of two models; a directional diatomic ordering which was proposed by Hoffmann and Kronmüller [67] and a magnetoelastic effect due to residual stress proposed by Herzer [28]. However, most of the work done by the researchers in recent times have been to establish or provide direct evidence to show that the stress induced anisotropy genuinely originates from one the two proposed models but, till date the origin is still not clear. This is because direct observations of structural anisotropy are lacking.

Ohnuma et al. [12] [38] suggested that the anisotropic energy originates from a magnetoelastic effect due to elastic elongation of the Fe-Si phase which has been constrained by a surrounding amorphous phase. Kraus et al. [36] suggested that the anisotropy is due to the inelastic polarization of the amorphous intergranular phase. Varga et al. [68] also suggested that the anisotropy was magnetoelastic in origin by relating the magnetic anisotropic energy to the magnetostriction of the crystalline phase. Fang et al. [69] suggested that the anisotropy is originated from the directional congregation of the agglomerated grains formed in stress annealed ribbon. Kernion et al. [70] also concluded that a magnetoelastic coupling is the primary source of the anisotropy. Fernandez et al. [71] found that the stress induced anisotropy is maximum when the annealing temperature is increased at constant applied stress. Lachowicz et al. [72] supported Hofmann's proposal because of the temperature dependence of the anisotropy. Nielsen et al. [73] suggested the stress induced anisotropy comprises of an inelastic part which is recovered post-annealing and a plastic part which remains post annealing.

In the magnetoelastic model, the induced anisotropy is transverse to the tensile stress direction and with increasing stress, the susceptibility of the linear magnetization curves decreases which implies that the $K_{u}$ increases. Per Equation (2.3), Herzer [28] suggested that the value of the $\lambda_{s}$ is a contribution from individual saturation magnetostriction coefficient values from the nanocrystalline and amorphous phases as shown in the expression below:

$$
\lambda_{s}=v_{c r} \lambda_{s}^{F e-S i}+\left(1-v_{c r}\right) \lambda_{s}^{A M}
$$

where $\lambda_{s}^{F e-S i}$ is the saturation magnetostriction coefficient of the $\alpha-\mathrm{Fe}(\mathrm{Si})$ nanocrystalline phase, $v_{c r}$ is the volume fraction of the crystalline phase and $\lambda_{s}^{A M}$ is the saturation magnetostriction coefficient of the amorphous phase. However, because of the inelastic creep deformation of the amorphous matrix with resulting bond-orientation state with no applied stress [74] yielding to internal stresses acting on the crystallites, the nature of $K_{u}$ would be a magnetoelastic anisotropy created in the Fe-Si grains. Consequently, the stress induced anisotropy is now related to the mechanical stress as:

$$
K_{u}=-\frac{3}{2} \sigma \lambda_{\mathrm{s}}^{\mathrm{Fe}-\mathrm{Si}}
$$


The two-phase microstructure of randomly oriented nanocrystals in a residual amorphous matrix in the nanocrystalline Fe-based alloys led to the suggestion of an atomic pair ordering model based on Neel's investigations [75] which suggested that the energy of each atomic pair in a solid solution was dependent on its relative orientation to the direction of uniaxial stress, such that if temperatures were high enough it would cause atomic rearrangement processes. This would lead to an anisotropic distribution of atomic pair axis which yields a magnetic anisotropy in the crystal. According to Neel's theory, the stress induced anisotropy is given by:

$$
K_{u}=\frac{33}{64} v \frac{c_{A}^{2} C_{B}^{2} L_{0} \sigma D_{0}}{C_{3} R T_{a} \kappa r_{0}}
$$

where $v$ is the volume fraction of the nanocrystals, $L_{0}=N_{A} l_{0}, l_{0}$ is the local anisotropy constant of one atomic pair and $N_{A}$ is Avogadro's constant, $D_{0} / r_{0}$ is the change in length correlated with one atomic rearrangement process related to average atomic distance, $c_{A}$ is the concentration of Fe in the FeSi nanocrystals, $C_{B}$ is the concentration of $\mathrm{Si}, C_{3}$ is the elastic constant, $\kappa$ is the compressibility, $T_{a}$ is the annealing temperature, and $\mathrm{R}$ is the gas constant. The atomic pair ordering model was shown to be negligible in contributing to the stress-induced magnetic anisotropy by Ohnuma et al. [11] concluding that that the induced magnetic anisotropy energy was well explained by residual strains and their magnetostrictions because structural anisotropy was observed in the lattice spacing of alloys with and without $\mathrm{Si}$.

Therefore, although the origin of the stress induced anisotropy has been narrowed down to two main models, most of the work done by researchers has further verified the magnetoelastic effect model and therefore looks likely to be the main origin of the anisotropy with negligible contributions from the atomic pair ordering. However, whether the induced anisotropy is dependent on creep (which is the overall plastic deformation) or the applied stress is still unanswered.

\section{Conclusions}

In summary, we can see that since the discovery of soft nanocrystalline alloys a lot of work has been done to understand the properties they possess, however, the arguments about the origin of stress induced anisotropy has been narrowed down to the magnetoelastic and atomic pair ordering models. Although the XRD, TEM and Mössbauer spectroscopy techniques have increased our understanding of the origin of induced magnetic anisotropy, details of the origin are still not clear. Therefore, efforts are/ and should be put in to improve the aspects of the induced magnetic anisotropy which is still under question. Some of the characterization techniques which should be used in addition to the commonly used transmission XRD, TEM and other methods such as; the electron holography which gives information about the microstructure of materials and can also reveal information about the domain structure and the synchrotron XRD which is a better and precise diffraction technique compared to the transmission XRD, giving accurate information about the kinetics of nanocrystallization, grain size 
and lattice parameters because data from synchrotron sources achieve better resolution. It is worth mentioning also that, in future studies about the structural origin of stress induced anisotropy, the study should be conducted in in-situ mode since it reveals real time information about the details of the experiment.

Whether the structural origin of stress induced anisotropy is due to the magnetoelastic coupling or the atomic pair ordering models, one thing certain is that, application of stress during annealing improves the soft magnetic properties of amorphous and nanocrystalline alloys. Therefore, with advances in experimental methods this enigma will soon be unraveled.

\section{Acknowledgements}

This work was supported by the Innovative group of the Zhejiang Solid State Photo-electronic Devices Laboratory, Shanghai Synchrotron Radiation Facility(SSRF) and Project 973 of the National Key Basic Research Program (No. 2012CB825705) of China.

\section{References}

[1] Askeland, D.K., Fulay, P.P. and Wright, W.J. (2010) The Science and Engineering of Materials. Cengage Learning, Inc., Boston.

[2] Callister Jr., W.D. (2007) Materials Science and Engineering-An Introduction. John Wiley \& Sons, Inc., Hoboken.

[3] Yoshizawa, Y., Oguma, S. and Yamauchi, K. (1988) New Fe-Based Soft Magnetic Alloys Composed of Ultrafine Grain Structure. Journal of Applied Physics, 64, 6044-6046. https://doi.org/10.1063/1.342149

[4] Herzer, G. (1989) Grain Structure and Magnetism of Nanocrystalline Ferromagnets. IEEE Transactions on Magnetics, 25, 3327-3329. https://doi.org/10.1109/20.42292

[5] Yoshizawa, Y. and Yamauchi, K. (1990) Fe-Based Soft Magnetic Alloys Composed of Ultrafine Grain Structure. Materials Transactions, IJM, 31, 307-314. https://doi.org/10.2320/matertrans1989.31.307

[6] Martienssen, W. and Warlimont, H. (2005) Springer Handbook of Condensed Matter and Materials Data. Springer, Berlin, Heidelberg.

https://doi.org/10.1007/3-540-30437-1

[7] Le Breton, J.M., Zorkovsk, A. and Kasiarova, M. (2004) Crystallization of $\mathrm{Fe}_{73.5} \mathrm{Cu}_{1^{-}}$ $\mathrm{RE}_{3} \mathrm{Si}_{13.5} \mathrm{~B}_{9}$ Ribbons with $\mathrm{RE}=\mathrm{Pr}, \mathrm{Nd}$, Gd. Journal of Physics. Condensed Matter, 16, 5555-5568. https://doi.org/10.1088/0953-8984/16/30/016

[8] Chau, N., Thanh, P.Q., Hoa, N.Q. and The, N.D. (2006) The Existence of Giant Magnetocaloric Effect and Laminar Structure in $\mathrm{Fe}_{73.5-\mathrm{x}} \mathrm{Cr}_{\mathrm{x}} \mathrm{Si}_{13.5} \mathrm{~B}_{9} \mathrm{Nb}_{3} \mathrm{Cu}_{1}$. Journal of Magnetism and Magnetic Materials, 304, 36-40.

[9] Zelenakova, A., Fuzer, J., Kollar, P., Kuzminski, M. and Svec, T. (2006) Magnetic Properties of Surface Layers of $\mathrm{Fe}_{71.5} \mathrm{Si}_{15.5-\mathrm{x}} \mathrm{Cu}_{1} \mathrm{Nb}_{3} \mathrm{~B}_{9} \mathrm{Al}_{\mathrm{x}}(\mathrm{x}=0,1,2,3$ at\%) Alloys with Substitutions by Aluminium. Journal of Magnetism and Magnetic Materials, 215, 459-462.

[10] Kurlyandskaya, G.V., Lukshina, V.A., Larranaga, A., Orue, I., Zaharova, A.A. and Shishkin, D.A. (2013) Induced Magnetic Anisotropy Features in $\mathrm{FeCrSiBNbCu}$ Nanocrystalline Alloy: Role of Stress Distribution Proven by Direct X-Ray Measurements. Journal of Alloys and Compounds, 566, 31-36.

[11] Ohnuma, M., Yanai, T., Hono, K., Nakano, M., Fukunaga, H., Yoshizawa, Y. and 
Herzer, G. (2010) Stress-Induced Magnetic and Structural Anisotropy of Nanocrystalline Fe-Based Alloys. Journal of Applied Physics, 108, Article ID: 093927. https://doi.org/10.1063/1.3506538

[12] Ohnuma, M., Yanai, T., Hono, K., Nakano, M., Fukunaga, H., Yoshizawa, Y. and Herzer, G. (2003) Direct Evidence for Structural Origin of Stress-Induced Magnetic Anisotropy in Fe-Si-B-Nb-Cu Nanocrystalline Alloys. Journal of Applied Physics, 83, 2859. https://doi.org/10.1063/1.1615672

[13] Hono, K., Ping, D.H., Ohnuma, M. and Onodera, H. (1999) Cu Clustering and Si Partitioning in the Early Crystallization Stage of an $\mathrm{Fe}_{73.5} \mathrm{Si}_{13.5} \mathrm{~B}_{9} \mathrm{Nb}_{3} \mathrm{Cu}_{1}$ Amorphous Alloy. Acta Materialia, 47, 997-1006.

[14] Cullity, B.D. and Graham, C.D. (2005) Introduction to Magnetic Materials. John Wiley \& Sons, Hoboken.

[15] Aharoni, A. (1996) Introduction to the Theory of Ferromagnetism. Clarendon Press.

[16] Chikazumi, S. (1997) Physics of Ferromagnetism. Clarendon Press.

[17] Donahue, M.J. and Porter, D.G. (2002) Analysis of Switching in Uniformly Magnetized Bodies. IEEE Transactions on Magnetics, 38, 2468-2470. https://doi.org/10.1109/TMAG.2002.803616

[18] Skumryev, V., Stoyanov, S., Zhang, Y., Hadjipanayis, G., Givord, D. and Nogués, J. (2003) Beating the Superparamagnetic Limit with Exchange Bias. Nature, 423, 850853. https://doi.org/10.1038/nature01687

[19] Meiklejohn, W.H. and Bean, C.P. (1957) New Magnetic Anisotropy. Physical Review, 105, 904-913. https://doi.org/10.1103/PhysRev.105.904

[20] Skomski, R. and Coey, J.M.D. (2016) Magnetic Anisotropy-How Much Is Enough for a Permanent Magnet? Scripta Materialia, 112, 3-8.

[21] Tracy, J.B., Weiss, D.N., Dinega, D.P. and Bawendi, M.G. (2005) Exchange Biasing and Magnetic Properties of Partially and Fully Oxidized Colloidal Nanoparticles. Physical Review B, 72, Article ID: 064404. https://doi.org/10.1103/PhysRevB.72.064404

[22] Murray, C.B., Sun, S., Doyle, H. and Betley, T. (2001) Monodisperse 3d Transition-Metal (Co, Ni, Fe) Nanoparticles and Their Assembly into Nanoparticle Superlattices. MRS Bulletin, 26, 985-991. https://doi.org/10.1557/mrs2001.254

[23] Chikazumi, S. and Graham, C. (1970) Magnetism and Metallurgy. Academic, New York, Vol. 2, 577.

[24] Srikala, D., Singh, V.N., Banerjee, A., Mehta, B.R. and Patnaik, S. (2008) Control of Magnetism in Cobalt Nanoparticles by Oxygen Passivation. The Journal of Physical Chemistry C, 112, 13882-13885. https://doi.org/10.1021/jp804086m

[25] Bozorth, R.M. (1934) Theory of the Heat Treatment of Magnetic Materials. Physical Review, 46, 232. https://doi.org/10.1103/PhysRev.46.232

[26] Parsons, R., Yanai, T., Kishimoto, H., Kato, A., Ohnuma, M. and Suzuki, K. (2015) Induced Magnetic Anisotropy in Si-Free Nanocrystalline Soft Magnetic Materials: A Transmission X-Ray Study. Journal of Applied Physics, 117, 17A333. https://doi.org/10.1063/1.4918785

[27] Alves, F., Desmoulins, J.B., HeHrisson, D., Rialland, J.F. and Costa, F. (2000) Stress-Induced Anisotropy in FINEMET- and Nanoperm-Type Crystalline Alloys Using Flash Annealing. Journal of Magnetism and Magnetic Materials, 34, 387-390.

[28] Herzer, G. (1994) Creep Induced Magnetic Anisotropy in Nanocrystalline Fe-CuNb-Si-B alloys. IEEE Transactions on Magnetics, 30, 4800-4802. https://doi.org/10.1109/20.334226 
[29] Csizmadia, E., Varga, L.K., Palanki, Z. and Zamborsky, F. (2015) Creep or Tensile Stress Induced Anisotropy FINEMET-Type Ribbons? Journal of Magnetism and Magnetic Materials, 374, 587-590.

[30] Snyder, R.L. (1992) In: Characterization of Materials. Lifshin, E. (Ed.). Vol. 2 A of Materials Science and Technology-A Comprehensive Treatment: Cahn, R.W., Haasen, P. and Kramer, E. (Eds.). Chapter 4. Weinheim: VCH.

[31] Liu, Y., Zhang, X. and Zhang, Z., Eds. (2001) Progress in Transmission Electron Microscopy. Tsinghua University Press and Springer, 273.

[32] Brundle, C.R., Evans, C.A. and Wilson, S., Eds. (1992) Encyclopedia of Materials Characterization-Surfaces, Interfaces, Thin Films. Elsevier, New York.

[33] Brandon, D.D. and Kaplan, W.D. (1999) Microstructural Characterization of Materials. Wiley, New York.

[34] Cahn, R., Ed. (2005) Concise Encyclopedia of Materials Characterization. 2nd Edition, Elsevier, San Diego, CA.

[35] Gavrilovic, A., Rafailovic, L.D., Minic, D.M., Wosik, J. and Angerer, P. (2011) Influence of Thermal Treatment on Structure Development and Mechanical Properties of Amorphous $\mathrm{Fe}_{73.5} \mathrm{Cu}_{1} \mathrm{Nb}_{3} \mathrm{Si}_{15.5} \mathrm{~B}_{7}$ Ribbon. Journal of Alloys and Compounds, 509, S119-S122.

[36] Kraus, L., Zaveta, K., Heczko, O., Duhaj, P., Vlasak, G. and Schnaider, T. (1992) Magnetic Anisotropy in As-Quenched and Stress-Annealed Amorphous and Nanocrystalline $\mathrm{Fe}_{73.5} \mathrm{Cu}_{1} \mathrm{RE}_{3} \mathrm{Si}_{13.5} \mathrm{~B}_{9}$ Alloys. Journal of Magnetism and Magnetic Materials, 112, 275-277.

[37] Gavrilovic, A., Minic, D.M., Rafailovic, L.D., Angerer, P., Wosik, J. and Maricic, A. (2010) Phase Transformations of $\mathrm{Fe}_{73.5} \mathrm{Cu}_{1} \mathrm{Nb}_{3} \mathrm{Si}_{15.5} \mathrm{~B}_{7}$ Amorphous Alloy upon Thermal Treatment. Journal of Alloys and Compounds, 504, 462-467.

[38] Ohnuma, M., Yanai, T., Hono, K., Nakano, M., Fukunaga, H. and Yoshizawa, Y. (2005) Origin of the Magnetic Anisotropy Induced by Stress Annealing in Fe-Based Nanocrystalline Alloy. Applied Physics Letters, 86, Article ID: 152513. https://doi.org/10.1063/1.1901807

[39] Ohnuma, M., Herzer, G., Kozikowski, P., Polak, C., Budinsky, V. and Koppoju, S. (2012) Structural Anisotropy of Amorphous Alloys with Creep-Induced Magnetic Anisotropy. Acta Materialia, 60, 1278-1286.

[40] Liu, Y., Zhen, M., Zeng, H. and Sellmyer, D.J. (2002) Handbook of Nanophase and Nanostructured Materials. Tsinghua University Press and Kluwer Academic Publishers.

[41] Miguel, C., Zhukov, A.P., del Val, J.J., Ramírez de Arellano, A. and González, J. (2005) Effect of Stress and/or Field Annealing on the Magnetic Behavior of the $\left(\mathrm{Co}_{77} \mathrm{Si}_{13.5} \mathrm{~B}_{9.5}\right)_{90} \mathrm{Fe}_{7} \mathrm{Nb}_{3}$ Amorphous Alloy. Journal of Applied Physics, 97, Article ID: 034911. https://doi.org/10.1063/1.1845577

[42] Serrano, I.G., Hernando, A. and Marín, P. (2014) Low Temperature Magnetic Behavior of Glass-Covered Magnetic Microwires with Gradient Nanocrystalline $\mathrm{Mi}$ crostructure. Journal of Applied Physics, 115, Article ID: 033903. https://doi.org/10.1063/1.4862540

[43] Greneche, J.M. and Miglierini, M. (1999) Mössbauer Spectrometry Applied to Iron-Based Nanocrystalline Alloys: I High Temperature Studies. In: Miglierini, M. and Petridis, D., Eds., Mössbauer Spectroscopy in Materials Science, Kluwer, Dordrecht, 243-256.

[44] Miglierini, M., Greneche, J.M. and Idzikowski, B. (2001) Temperature Mössbauer Effect Study of Nanocrystalline FeMCuB Alloys. Materials Science and Engineering: 
$A, 304,937-940$.

[45] Clavaguera, N., Pradell, T., Jie, Z. and Clavaguera-Mora, M.T. (1995) A Mössbauer Study of the Nanocrystallization Process in $\mathrm{Fe}_{73.5} \mathrm{Cu}_{1} \mathrm{Nb}_{3} \mathrm{Si}_{17.5} \mathrm{~B}_{5}$ Alloy. Nanostructured Materials, 6, 453-456.

[46] Lukshin,a V.A., Serikov, V.V., Kleinerman, N.M., Dmitrieva, N.V., Volkova, E.G. and Potapov, A.P. (2007) Induced Magnetic Anisotropy and Structure of Fe-Based Nanocrystalline Material with Different Si and Co Content. Journal of Magnetism and Magnetic Materials, 316, 816-819.

[47] He, K.-Y. (2011) Optimum Condition for Magnetic Properties of Two-Phase Soft Magnetic Alloys. Journal of Applied Physics, 110, Article ID: 043925. https://doi.org/10.1063/1.3622583

[48] Kane, S.N., Ruwali, K., Gupta, A., Kraus, L. and Haslar, V. (1996) Creep Induced Magnetic Anisotropy in Amorphous $\mathrm{Fe}_{25} \mathrm{Co}_{60} \mathrm{~B}_{15}$. Journal of Magnetism and Magnetic Materials, 157-158, 519-520.

[49] Miglierini, M. and Lipka, J. (1995) Mössbauer Study of FeCuNbSiB-Type Nanocrystals. Journal of Magnetism and Magnetic Materials, 140-144, 463-464.

[50] Gupta, P., Gupta, A., Shukla, A., Ganguli, T., Sinha, A.K., Principi, G. and Maddalena, A. (2011) Structural Evolution and the Kinetics of Clustering in the Amorphous Phase of Fe-Cu-Nb-Si-B Alloy. Journal of Applied Physics, 110, Article ID: 033537. https://doi.org/10.1063/1.3622325

[51] Hasiak, M., Ciurzynska, W.H. and Yamashiro, Y. (2000) Microstructure and Some Magnetic Properties of Amorphous and Nanocrystalline Fe-Cu-Nb-Si-B Alloys. Materials Science and Engineering: A, 293, 261-266.

[52] Yoshizawa, Y., Fujii, S., Ping, D.H., Ohnuma, M. and Hono, K. (2003) Magnetic Properties of Nanocrystalline FeMCuNbSiB Alloys (M: Co, Ni). Scripta Materialia, 48, 863-868.

[53] Ohnuma, M., Ping, D.H., Abe, T., Onodera, H., Hono, K. and Yoshizawa, Y. (2003) Optimization of the Microstructure and Properties of Co-Substituted Fe-Si-B-Cu$\mathrm{Nb}$ Nanocrystalline Soft Magnetic Alloys. Journal of Applied Physics, 93, 91869194. https://doi.org/10.1063/1.1569396

[54] Hilzinger, R. and Rodewald, W. (2012) Magnetic Materials. Publicis Publishing, Erlangen.

[55] Murillo, N., Gonzalez, J., Blanco, J.M. and Vazquez, M. (1993) Stress Induced Anisotropy and Temperature Dependence of the Magnetostriction in $\mathrm{Fe}_{73.5} \mathrm{Cu}_{1} \mathrm{Nb}_{3} \mathrm{Si}_{13.5} \mathrm{~B}_{9}$ Amorphous Alloy. Journal of Applied Physics, 74, 3323. https://doi.org/10.1063/1.354556

[56] Hofmann, B. and Kronmüller, H. (1995) Creep Induced Magnetic Anisotropy in Fe-Cu-Nb-Si-B. Nanostructured Materials, 6, 961-964.

[57] Reininger, Th., Hofmann, B. and Kronmüller, H. (1992) Magnetization processes in Nanocrystalline Ferromagnets. Journal of Magnetism and Magnetic Materials, 111, 220-224.

[58] Ohnuma, M., Hono, K., Linderoth, S., Pedersen, J.S., Yoshizawa, Y. and Onodera, H. (2000) Small Angle Neutron Scattering and Differential Scanning Calorimetry Studies on the Copper Clustering Stage of Fe-Nb-Si-B-Cu Nanocrystalline Alloys. Acta Materialia, 48, 4783-4790.

[59] Chen, Y.M., Ohkubo, T., Ohta, M., Yoshizawa, Y. and Hono, K. (2009) 3D Atom Probe Analysis of Fe-B Based Nanocrystalline Soft Magnetic Materials. Acta Materialia, 57, 4463-4472.

[60] Makino, A., Men, H., Kubota, T. and Inoue, A. (2009) New Fe-Metalloids Based 
Nanocrystalline Alloys with High $B_{s}$ of $1.9 \mathrm{~T}$ and Excellent Magnetic Softness. Journal of Applied Physics, 105, 07A308.

[61] Hono, K., Hiraga, K., Wang, Q., Inuoe, A. and Sakurai, T. (1992) The Microstructure Evolution of a $\mathrm{Fe}_{73.5} \mathrm{Cu}_{1} \mathrm{Nb}_{3} \mathrm{Si}_{13.5} \mathrm{~B}_{9}$ Nanocrystalline Soft Material. Acta Metallurgica et Materialia, 40, 2137-2147.

[62] Graf, T., Hampel, G. and Korus, J. (1995) Influence of Nb Concentration on Structure and Crystallization Onset of Amorphous $\mathrm{Fe}(\mathrm{CuNb}) \mathrm{SiB}$. Nanostructured Materials, 6, 469-472.

[63] Hermann, H., Heinemann, A., Mattern, N. and Weidenmann, A. (2000) Experimental Evidence for Inhibitor-Controlled Mechanism of Nanocrystallization in Amorphous Metallic Alloys. Europhysics Letters, 51, 127-132. https://doi.org/10.1209/epl/i2000-00520-y

[64] Ayers, J.D., Harris, V.G., Sprague, J.A. and Elam, W.T. (1994) On the Role of Cu and $\mathrm{Nb}$ in the Formation of Nanocrystals in Amorphous $\mathrm{Fe}_{73.5} \mathrm{Cu}_{1} \mathrm{Nb}_{3} \mathrm{Si}_{13.5} \mathrm{~B}_{9} . A p$ plied Physics Letters, 64, 974-976. https://doi.org/10.1063/1.110923

[65] Pundt, A., Humpel, G. and Hesse, J. (1992) Mössbauer Effect Studies on Amorphous and Nanocrystalline $\mathrm{Fe}_{73.5} \mathrm{Cu}_{1} \mathrm{Nb}_{3} \mathrm{Si}_{13.5} \mathrm{~B}_{9}$. Zeitschrift für Physik $B$ Condensed Matter, 87, 65-72.

[66] Müller, M. and Mattern, N. (1994) The Influence of Refractory Element Additions on the Magnetic Properties and on the Crystallization Behavior of Nanocrystalline Soft Magnetic Fe-B-Si-Cu Alloys. Journal of Magnetism and Magnetic Materials, 136, 79-87.

[67] Hofmann, B. and Kronmüller, H. (1996) Stress-Induced Magnetic Anisotropy in Nanocrystalline FeCuNbSiB Alloy. Journal of Magnetism and Magnetic Materials, $152,91-98$.

[68] Varga L.K., Gercsi, Zs., Kovacs, Gy., Kakay, A. and Mazaleyrat, F. (2003) StressInduced Magnetic Anisotropy in Nanocrystalline Alloys. Journal of Magnetism and Magnetic Materials, 254-255, 477-479.

[69] Fang, Y.Z., Zheng, J.J., Wu, F.M., Xu, Q.M., Zhang, J.Q., Ye, H.Q., Zheng, J.L. and Li, T.Y. (2010) Mesostructural Origin of Stress-Induced Magnetic Anisotropy in Fe-Based Nanocrystalline Ribbons. Applied Physics Letters, 96, Article ID: 092508. https://doi.org/10.1063/1.3330931

[70] Kernion, S.J., Ohodnicki, P.R., Grossmann, J., Leary, A., Shen, S., Keylin, V., Huth, J.F., Horwath, J., Lucas, M.S. and McHenry, M.E. (2012) Giant Induced Magnetic Anisotropy in Strain Annealed Co-Based Nanocomposite Alloys. Applied Physics Letters, 101, Article ID: 102408. https://doi.org/10.1063/1.4751253

[71] Fernandez-Gubieda, M.L., Barandiaran, J.M. and Nielsen, O.V. (1987) Simultaneous Observation of Viscoelastic Deformation and Induced Magnetic Anisotropy in $\left[\mathrm{Co}_{1-\mathrm{x}}(\mathrm{FeNi})_{\mathrm{x}}\right]_{75} \mathrm{Si}_{15} \mathrm{~B}_{10}$ Metallic Glasses. Journal of Applied Physics, 62, 2579. https://doi.org/10.1063/1.339432

[72] Lachowicz, H.K., Neuweiler, A., Poplawski, F. and Dynowska, E. (1997) On the Origin of Stress-Anneal-Induced Anisotropy in FINEMET-Type Nanocrystalline Magnets. Journal of Magnetism and Magnetic Materials, 173, 287-294.

[73] Nielsen, O.V. (1983) Separation into Two Contributions of Stress Anneal Induced Magnetic Anisotropy in Metallic Glass Ribbons. Journal of Magnetism and Magnetic Materials, 36, 81-85.

[74] Suzuki, Y., Haimovich, J. and Egami, T. (1987) Bond-Orientational Anisotropy in Metallic Glasses Observed by X-Ray Diffraction. Physical Review B, 35, 2162. https://doi.org/10.1103/PhysRevB.35.2162 
[75] Neél, M.L. (1954) Magnetic Surface Anisotropy and Superlattice Formation by Orientation. Journal de Physique et Le Radium, 15, 225-239.

https://doi.org/10.1051/jphysrad:01954001504022500

Submit or recommend next manuscript to SCIRP and we will provide best service for you:

Accepting pre-submission inquiries through Email, Facebook, LinkedIn, Twitter, etc. A wide selection of journals (inclusive of 9 subjects, more than 200 journals)

Providing 24-hour high-quality service

User-friendly online submission system

Fair and swift peer-review system

Efficient typesetting and proofreading procedure

Display of the result of downloads and visits, as well as the number of cited articles Maximum dissemination of your research work

Submit your manuscript at: http://papersubmission.scirp.org/

Or contact jemaa@scirp.org 